\title{
An improved routing mechanism using bio-inspired for energy balancing in wireless sensor networks
}

\begin{abstract}
Planning of energy-efficient protocols is critical for wireless sensor networks (WSNs) because of the constraints on sensor node's energy. Therefore, the routing protocol should be able to achieve uniform power dissipation during transmission to the sink node. In this paper, we present a self-optimization scheme for WSN which is able to utilize and optimize the sensor nodes' resources, especially the batteries, to achieve balanced energy consumption across all sensors. This method is based on Ant Colony Optimization (ACO) meta heuristic which is adopted to enhance the paths with the best quality function. The assessment of this function depends on multi-criteria metrics such as the minimum residual battery power, hop numbers and average energy of both route and network. This method also distribute the traffic load of sensor nodes throughout the WSN leading to reduced energy usage, extended network life time and reduced packet loss. Simulation results show that our scheme performs much better than Energy Efficient Ant-Based Routing (EEABR) in terms of energy consumption and efficiency.
\end{abstract}

Keyword: Energy balance; WSN; Ant colony optimization; Energy conservation 